\title{
Onsager Consistency Checks for Multicomponent Diffusion Models
}

\author{
JOHN M. ZIELINSKI, ${ }^{1}$ SACIDE ALSOY ${ }^{2}$ \\ ${ }^{1}$ Air Products \& Chemicals, Incorporated, 7201 Hamilton Boulevard, Allentown, Pennsylvania 18195 \\ ${ }^{2}$ Izmir Institute of Technology, Muhendislik Fakultesi, Gaziosmanpasa Bulvari, No. 16, 35230, Cankaya-Izmir, Turkey
}

Received 17 November 2000; revised 2 April 2001; accepted 10 April 2001

\begin{abstract}
The Onsager reciprocity relations are applied to several recently proposed multicomponent diffusion models in an attempt to gauge their validity and ascertain their applicability. Each of these friction-based diffusion models stems from the more general Bearman formalism through various assumptions regarding the individual friction coefficients. By assessing the compliance of the Bearman model with respect to the Onsager relations, we ascertain the validity of the simplifications introduced to each diffusion model and suggest which postulates lead to results consistent with the Onsager relations. Although some models are not consistent with the Onsager relations, each model predicts the multicomponent drying of polymer films reasonably well. The necessity for consistency with the Onsager development is, therefore, revisited. (C) 2001 John Wiley \& Sons, Inc. J Polym Sci Part B: Polym Phys 39: 1496-1504, 2001

Keywords: self-diffusion; mutual diffusion; multicomponent diffusion; cross coefficients; friction coefficients; transport; Onsager; drying
\end{abstract}

\section{INTRODUCTION}

The drying of solvent-coated polymer films and the formation of asymmetric polymer membranes through controlled coagulation are key steps in the economic production of many products formed from multicomponent transport and phase equilibria considerations. ${ }^{1-3}$ Typical products include photographic film, synthetic fibers, adhesives, and hollow-fiber membranes. Multicomponent diffusion models are consequently sought to accurately describe the temperature and concentration dependence of diffusional fluxes during the processing of these products. ${ }^{3-5}$

The authors are pleased to dedicate this article to Professor Hans Sillescu in honor of his 65th birthday.

Correspondence to: J. M. Zielinski (E-mail: zielinjm@apci. com)

Journal of Polymer Science: Part B: Polymer Physics, Vol. 39, 1496-1504 (2001) (c) 2001 John Wiley \& Sons, Inc.
An analysis of a multicomponent diffusion process involves quantification of an array of diffusion coefficients that describes how the flux of one component is influenced by its own concentration gradient and the concentration gradient of every other component in the system. ${ }^{6-9}$ The number of diffusion coefficients $\left(D_{i j}\right)$ employed to describe transport within a system of $n$ components is equal to $(n-1)^{2}$. The diagonal terms of the array $\left(D_{i i}\right)$ are called the main or principal diffusion coefficients, whereas the off-diagonal terms $\left(D_{i j}\right.$, where $i \neq j$ ) are termed the cross coefficients.

On the basis of the Onsager reciprocal relations ${ }^{10}$ the diffusion coefficient matrix is subject to certain constraints that serve to reduce the number of independent diffusion coefficients in an $n$-component system to $n(n-1) / 2$. Few experimental studies, however, have attempted to independently measure the principal and cross diffusion coefficients and assess their compliance with the Onsager relations. ${ }^{11,12}$ In general, the princi- 
pal and cross diffusion coefficients are determined independently without the application of the constraints ascribed by Onsager. Alternatively, the cross diffusion coefficients may be simply presumed to be negligible, in which case only effective principal diffusion coefficients are measured.

Although multicomponent thermodynamic data and models can be stringently assessed for consistency by means of the Gibbs-Duhem relation, the only criteria that currently exist to ascertain consistency in multicomponent diffusion analyses are the Onsager relations, the absolute rigor of which has yet to be indisputably established. Although strict adherence of equilibrium and transport models to the Gibbs-Duhem and Onsager relations is deemed essential for fundamental consistency, practical engineering tools often do not conform to these relations. Insight into the development of new models may be gleaned by an examination of how other models fail their respective consistency tests while retaining accurate predictive capability.

Several friction-based multicomponent diffusion models have been recently suggested without an accompanying analysis for Onsager consistency. Each of these models stems from the more general Bearman ${ }^{13}$ formalism through assumptions regarding the individual friction coefficients. In this work, we examine the simplifications introduced in the development of these diffusion models and examine the implications of these assumptions and their consistency with the Onsager relations.

\section{THEORY}

Derivation of the Onsager consistency relations (OCRs) for multicomponent diffusion requires the application of three basic postulates: (1) thermodynamic variables such as entropy, chemical potential, and temperature can be correctly defined in a differential volume of a system that is not at equilibrium; (2) a linear relationship exists between forces and fluxes; and (3) the linear cross coefficients are symmetric. ${ }^{6,10,14,15}$ Little guidance, however, is offered regarding the appropriate selection of forces and fluxes. Because most theoretical transport models and experimental diffusion measurements are based on a volume frame of reference, the following expression is often considered to be the most appropriate starting point for establishing the OCRs:

$$
\sigma=-\frac{1}{T} \sum_{i=1}^{n} j_{i}^{\neq} \nabla \hat{\mu}_{i}
$$

where $\sigma$, the rate of entropy production in units of energy/volume-time-temperature, is expressed in terms of the mass flux relative to the volumeaverage velocity $\left(j_{i}^{*}\right)$ and the specific chemical potential gradient $\left(\nabla \hat{\mu}_{i}\right)$ given in energy/massdistance. $T$ represents the absolute temperature.

Consideration of the Gibbs-Duhem relation and the definition of the mass flux with respect to the volume-average velocity renders constraints to eq 1 . These constraints can be written as

$$
\begin{aligned}
& \sum_{i=1}^{n} \rho_{i} \nabla \hat{\mu}_{i}=0 \\
& \sum_{i=1}^{n} \hat{V}_{i} j_{i}^{\neq}=0
\end{aligned}
$$

Here, $\rho_{i}$ and $\hat{V}_{i}$ are the mass concentration and partial specific volume, respectively, of component $i$.

The substitution of eqs 2 and 3 into eq 1 and the realization that the product of $\rho_{i}$ and $\hat{V}_{i}$ equals the volume fraction of component $i\left(\phi_{i}\right)$ yield the expressions often cited as the initial point for the development of the OCRs:

$$
\sigma=-\frac{1}{T} \sum_{i=1}^{n-1} j_{i}^{\neq} \hat{X}_{i}
$$

where

$$
\begin{gathered}
\hat{X}_{i}=\sum_{j=1}^{n-1} \alpha_{i j} \nabla \hat{\mu}_{j} \\
\alpha_{i j}=\delta_{i j}+\frac{\rho_{j} \hat{V}_{i}}{\phi_{n}}
\end{gathered}
$$

and $\delta_{i j}$ is the Kronecker $\delta$ function.

The concept of entropy production (eq 4) is typically not again discussed in the subsequent development of the OCRs. In fact, the principal reason to rearrange eqs $1-3$ to obtain eqs $4-6$ is to provide a definition of force $\left(\hat{X}_{j}\right)$ to which one can apply postulate 2 . This result and the fact that the thermodynamic flux is not written as the 
time rate of change of the state of the system are the two primary reasons the Onsager relations have been severely criticized in the literature. ${ }^{16,17}$

The linear relationship between forces and fluxes in the OCR is, therefore, given as

$$
-j_{i}^{\neq}=\sum_{j=1}^{n-1} L_{i j} \hat{X}_{i}
$$

where $L_{i j}$ denotes the linear coefficients identified in postulate 2 that relate forces to fluxes. Furthermore, if postulate 3 regarding symmetry is accepted, then the off-diagonal coefficients must be equal; that is, $L_{i j}=L_{j i}$ when $i \neq j$. For the sake of brevity and applicability to the analysis specifically provided here, we now develop the OCR for a ternary system. The application of eqs 1-6 to higher order multicomponent systems is straightforward and is left to the interested reader.

By expanding eq 7 and implementing the terms defined in eqs $5-6$, one obtains the following expressions for the mass flux of components 1 and 2 in a ternary mixture:

$$
\begin{aligned}
j_{1}^{\neq}= & -\left[\left(L_{11}\left(1+\frac{\phi_{1}}{\phi_{3}}\right)+L_{12}\left(\frac{\rho_{1} \hat{V}_{2}}{\phi_{3}}\right)\right) \frac{\partial \hat{\mu}_{1}}{\partial \rho_{1}}\right. \\
& \left.+\left(L_{11}\left(\frac{\rho_{2} \hat{V}_{1}}{\phi_{3}}\right)+L_{12}\left(1+\frac{\phi_{1}}{\phi_{3}}\right)\right) \frac{\partial \hat{\mu}_{2}}{\partial \rho_{1}}\right] \nabla \rho_{1} \\
& -\left[\left(L_{11}\left(1+\frac{\phi_{1}}{\phi_{3}}\right)+L_{12}\left(\frac{\rho_{1} \hat{V}_{2}}{\phi_{3}}\right)\right) \frac{\partial \hat{\mu}_{1}}{\partial \rho_{2}}\right. \\
& \left.+\left(L_{11}\left(\frac{\rho_{2} \hat{V}_{1}}{\phi_{3}}\right)+L_{12}\left(1+\frac{\phi_{1}}{\phi_{3}}\right)\right) \frac{\partial \hat{\mu}_{2}}{\partial \rho_{2}}\right] \nabla \rho_{2} \\
j_{2}^{\neq}= & -\left[\left(L_{21}\left(1+\frac{\phi_{1}}{\phi_{3}}\right)+L_{22}\left(\frac{\rho_{1} \hat{V}_{2}}{\phi_{3}}\right)\right) \frac{\partial \hat{\mu}_{1}}{\partial \rho_{1}}\right. \\
& \left.+\left(L_{21}\left(\frac{\rho_{2} \hat{V}_{1}}{\phi_{3}}\right)+L_{22}\left(1+\frac{\phi_{1}}{\phi_{3}}\right)\right) \frac{\partial \hat{\mu}_{2}}{\partial \rho_{1}}\right] \nabla \rho_{1} \\
& -\left[\left(L_{21}\left(1+\frac{\phi_{1}}{\phi_{3}}\right)+L_{22}\left(\frac{\rho_{1} \hat{V}_{2}}{\phi_{3}}\right)\right) \frac{\partial \hat{\mu}_{1}}{\partial \rho_{2}}\right. \\
& \left.+\left(L_{21}\left(\frac{\rho_{2} \hat{V}_{1}}{\phi_{3}}\right)+L_{22}\left(1+\frac{\phi_{1}}{\phi_{3}}\right)\right) \frac{\partial \hat{\mu}_{2}}{\partial \rho_{2}}\right] \nabla \rho_{2}
\end{aligned}
$$

A comparison of eqs 8 and 9 to the Fickian transport equations for components 1 and 2

$$
j_{1}^{\neq}=-D_{11} \nabla \rho_{1}-D_{12} \nabla \rho_{2}
$$

$$
j_{2}^{\neq}=-D_{21} \nabla \rho_{1}-D_{22} \nabla \rho_{2}
$$

immediately yields expressions for the diffusion coefficient matrix in terms of the Onsager mobility coefficients:

$$
\begin{aligned}
D_{11}= & {\left[L_{11}\left(1+\frac{\phi_{1}}{\phi_{3}}\right)+L_{12}\left(\frac{\rho_{1} \hat{V}_{2}}{\phi_{3}}\right)\right] \frac{\partial \hat{\mu}_{1}}{\partial \rho_{1}} } \\
+ & {\left[L_{11}\left(\frac{\rho_{2} \hat{V}_{1}}{\phi_{3}}\right)+L_{12}\left(1+\frac{\phi_{2}}{\phi_{3}}\right)\right] \frac{\partial \hat{\mu}_{2}}{\partial \rho_{1}} } \\
D_{12}= & {\left[L_{11}\left(1+\frac{\phi_{1}}{\phi_{3}}\right)+L_{12}\left(\frac{\rho_{1} \hat{V}_{2}}{\phi_{3}}\right)\right] \frac{\partial \hat{\mu}_{1}}{\partial \rho_{2}} } \\
+ & {\left[L_{11}\left(\frac{\rho_{2} \hat{V}_{1}}{\phi_{3}}\right)+L_{12}\left(1+\frac{\phi_{2}}{\phi_{3}}\right)\right] \frac{\partial \hat{\mu}_{2}}{\partial \rho_{2}} } \\
D_{21}= & {\left[L_{21}\left(1+\frac{\phi_{1}}{\phi_{3}}\right)+L_{22}\left(\frac{\rho_{1} \hat{V}_{2}}{\phi_{3}}\right)\right] \frac{\partial \hat{\mu}_{1}}{\partial \rho_{1}} } \\
+ & {\left[L_{21}\left(\frac{\rho_{2} \hat{V}_{1}}{\phi_{3}}\right)+L_{22}\left(1+\frac{\phi_{2}}{\phi_{3}}\right)\right] \frac{\partial \hat{\mu}_{2}}{\partial \rho_{1}} } \\
D_{22}= & {\left[L_{21}\left(1+\frac{\phi_{1}}{\phi_{3}}\right)+L_{22}\left(\frac{\rho_{1} \hat{V}_{2}}{\phi_{3}}\right)\right] \frac{\partial \hat{\mu}_{1}}{\partial \rho_{2}} } \\
+ & {\left[L_{21}\left(\frac{\rho_{2} \hat{V}_{1}}{\phi_{3}}\right)+L_{22}\left(1+\frac{\phi_{2}}{\phi_{3}}\right)\right] \frac{\partial \hat{\mu}_{2}}{\partial \rho_{2}} }
\end{aligned}
$$

Existing multicomponent diffusion models exhibit the common feature that, for ternary systems, the principal and cross diffusion coefficients can be cast into the generic forms shown in eqs 16-19:

$$
\begin{aligned}
& D_{11}=A_{11} \frac{\partial \hat{\mu}_{1}}{\partial \rho_{1}}+B_{11} \frac{\partial \hat{\mu}_{2}}{\partial \rho_{1}} \\
& D_{12}=A_{12} \frac{\partial \hat{\mu}_{1}}{\partial \rho_{2}}+B_{12} \frac{\partial \hat{\mu}_{2}}{\partial \rho_{2}} \\
& D_{21}=A_{21} \frac{\partial \hat{\mu}_{1}}{\partial \rho_{1}}+B_{21} \frac{\partial \hat{\mu}_{2}}{\partial \rho_{1}} \\
& D_{22}=A_{22} \frac{\partial \hat{\mu}_{1}}{\partial \rho_{2}}+B_{22} \frac{\partial \hat{\mu}_{2}}{\partial \rho_{2}}
\end{aligned}
$$

where the prefactors $A_{i j}$ and $B_{i j}$ represent a variety of expressions for the assorted multicomponent diffusion models available in the literature. 
The combination of eqs $12-15$ and $16-19$ reveals that constraints are imposed on the expressions for each $A_{i j}$ and $B_{i j}$. These constraints provide a first-pass screening with regard to the consistency of multicomponent diffusion models relative to Onsager reciprocal relations:

$$
\begin{aligned}
& A_{11}=A_{12}=L_{11}\left[1+\frac{\phi_{1}}{\phi_{3}}\right]+L_{12}\left[\frac{\rho_{1} \hat{V}_{2}}{\phi_{3}}\right] \\
& B_{11}=B_{12}=L_{11}\left[\frac{\rho_{2} \hat{V}_{1}}{\phi_{3}}\right]+L_{12}\left[1+\frac{\phi_{2}}{\phi_{3}}\right] \\
& A_{21}=A_{22}=L_{21}\left[1+\frac{\phi_{1}}{\phi_{3}}\right]+L_{22}\left[\frac{\rho_{1} \hat{V}_{2}}{\phi_{3}}\right] \\
& B_{21}=B_{22}=L_{21}\left[\frac{\rho_{2} \hat{V}_{1}}{\phi_{3}}\right]+L_{22}\left[1+\frac{\phi_{2}}{\phi_{3}}\right]
\end{aligned}
$$

The algebraic manipulation of eqs $20-23$ yields the cross (phenomenological) coefficients, $L_{12}$ and $L_{21}$, which may be written explicitly as

$$
\begin{aligned}
& L_{12}=B_{11} \phi_{3}\left[1+\frac{\phi_{1}}{\phi_{3}}\right]-A_{11} \phi_{3}\left[\frac{\rho_{2} \hat{V}_{1}}{\phi_{3}}\right] \\
& L_{21}=A_{22} \phi_{3}\left[1+\frac{\phi_{2}}{\phi_{3}}\right]-B_{22} \phi_{3}\left[\frac{\rho_{1} \hat{V}_{2}}{\phi_{3}}\right]
\end{aligned}
$$

Because $L_{12}=L_{21}$ by symmetry, eqs 24 and 25 together establish an essential condition for the consistency of multicomponent diffusion models with respect to the Onsager relations. In the next section, we apply this consistency check to several recently proposed diffusion models ${ }^{18,19}$ and the Bearman formalism, ${ }^{13}$ from which all known friction-based diffusion models are derived.

\section{RESULTS AND DISCUSSION}

The Bearman statistical mechanical theory ${ }^{13}$ contends that, when a binary diffusion process is unaffected by pressure $(P)$ gradients and temperature $(T)$ is constant, the mutual diffusion and self-diffusion coefficients within the system can be related to the friction coefficients describing the interactions between each of the components. Several practical diffusion models ${ }^{18,19}$ have been recently proposed to predict both the main- and cross diffusion coefficients in multicomponent systems. Each of these formalisms, however, can be shown to originate from the more general Bearman statistical mechanical theory through assumptions regarding the frictional coefficients. We examine the consistency of each of these models with respect to the Onsager relations by inserting the corresponding $A_{i j}$ and $B_{i j}$, which are defined in Table I, into eqs 24 and 25 and ascertaining if resulting expressions are equal.

\section{Bearman (1961)}

The model proposed by Bearman is grounded in statistical mechanical theory and relates mutual diffusion coefficients to self-diffusivities through friction coefficients. The well-known HartleyCrank $^{20}$ and Darken ${ }^{21}$ equations, which are generally the starting point to relate self-diffusion and mutual diffusion coefficients in binary (e.g., gas/solid) systems, constitute a subset of the more general Bearman model. These models assume regular solution behavior (in the Bearman sense) and a geometric mean relationship between the friction coefficients. The geometric mean approximation has been shown to qualitatively represent transport in binary systems but not to be always quantitatively accurate. ${ }^{22-24}$ The random phase approximation, sometimes used to model polymer-polymer interdiffusion, also stems from the Bearman model by employing an algebraic mean approximation between friction coefficients. $^{25}$

The Bearman model satisfies the $\mathrm{OCRs}^{3}$ and provides a standard to which other models can be compared. Practical usage of this model, however, is limited because the dependence of the friction coefficients on temperature and concentration is presently unknown. Extensions to the Bearman formalism have also been proposed. ${ }^{26}$

\section{Zielinski-Hanley (1999)}

This model is one of the few predictive multicomponent models in the literature that relates all the coefficients in a diffusion matrix to measurable self-diffusion and thermodynamic data. Although the Bearman model ${ }^{13}$ was not considered in its development, the Zielinski-Hanley model ${ }^{18}$ can be shown to be a subset of the more general Bearman formalism if we assume that the friction coefficients are related to each other through ratios of the species molecular weights. In a ternary system, this amounts to 


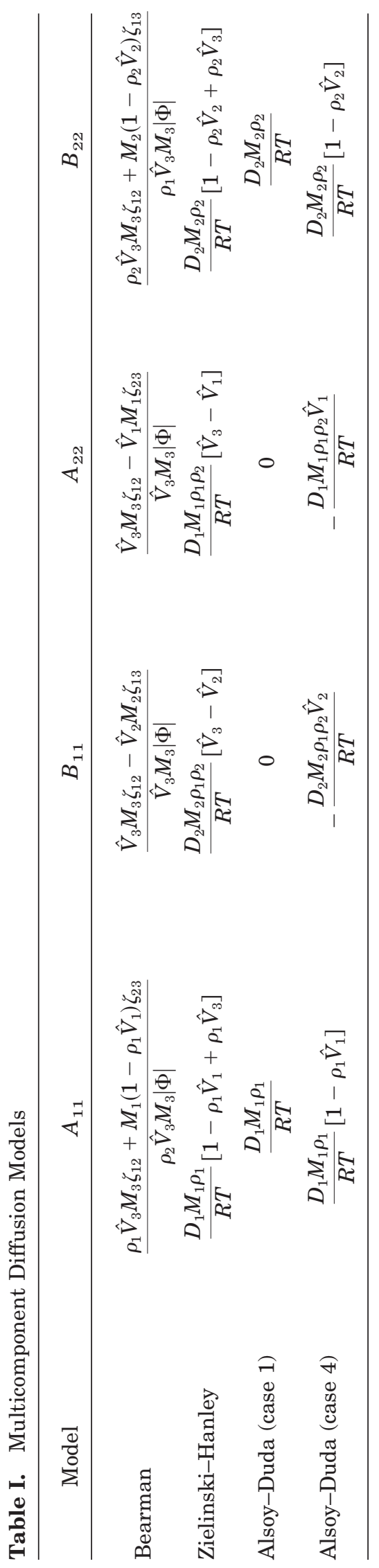

$$
\frac{\zeta_{12}}{\zeta_{13}}=\frac{M_{2}}{M_{3}}
$$

When Onsager consistency is applied to the ternary form of this model, a relationship between the self-diffusion coefficients emerges:

$$
\frac{D_{1}}{D_{2}}=\frac{M_{2}}{M_{1}}
$$

Although this relationship may approximately be true for mixtures consisting exclusively of low molecular weight species, it is not expected to be generally valid. Consequently, the Zielinski-Hanley model fails the Onsager consistency test.

For the sake of comparison, an analogous expression for $\zeta_{12} / \zeta_{13}$ in the Bearman framework is

$$
\frac{\zeta_{12}}{\zeta_{13}}=\frac{M_{2} R T}{\rho_{2} N_{A}^{2} \zeta_{13}}\left[1-\frac{D_{1} \rho_{1} \zeta_{11} N_{A}^{2}}{M_{1} R T}\right]-\frac{M_{2} \rho_{3}}{M_{3} \rho_{2}}
$$

\section{Alsoy-Duda (1999)}

In their effort to model the drying of polymer films, Alsoy and Duda ${ }^{19}$ proposed four models (cases 1-4) that can represent multicomponent transport processes reasonably well. Each case is derived from the Bearman statistical mechanical theory. Cases 1 and 4 provide functional forms for each component in the diffusion coefficient matrix $\left(D_{i j}\right)$, whereas cases 2 and 3 assert that the cross coefficients are negligible. Under some conditions, neglecting the cross coefficients is not expected to introduce significant error in the resulting flux predictions. From a theoretical standpoint, however, these models clearly cannot satisfy the Onsager relations. In the following discussion, therefore, we consider only cases 1 and 4 .

\section{Case 1}

In this first case, the ratio of friction factors between components $i$ and $k\left(\zeta_{i k}\right)$ and $j$ and $k\left(\zeta_{j k}\right)$ is assumed to be constant and equal to the ratio of the partial molar volumes of components $i$ and $j$; therefore,

$$
\frac{\zeta_{i k}}{\zeta_{j k}}=\frac{\tilde{V}_{i}}{\tilde{V}_{j}}
$$


Implementing this criterion into the OCRs yields a result similar to that derived for the ZielinskiHanley model:

$$
\frac{D_{1}}{D_{2}}=\frac{\tilde{V}_{2}}{\tilde{V}_{1}}
$$

where the molecular weight is replaced by the partial molar volume. As with the Zielinski-Hanley model, this relationship is not expected to be universally true, in which case this formalism fails the Onsager consistency check.

\section{Case 4}

This model is very similar in functional form to the Zielinski-Hanley model (see Table I). Its predictive capability, however, can be appreciably different. The case 4 model assumes that the friction coefficients among all of the solute molecules are identically equal to zero (i.e., $\zeta_{11}=\zeta_{22}=\zeta_{12}$ $=0)$. This assumption imposes a relationship between friction factors $\zeta_{13}$ and $\zeta_{23}$. From the Bearman model, the ratio of $\zeta_{13}$ to $\zeta_{23}$ may be written as

$$
\frac{\zeta_{13}}{\zeta_{23}}=\frac{\frac{R T}{N_{A}^{2} D_{1}}\left[1-\frac{D_{1} \rho_{2} \zeta_{11} N_{A}^{2}}{M_{1} R T}\right]-\frac{\rho_{2} \zeta_{12}}{M_{2}}}{\frac{R T}{N_{A}^{2} D_{2}}\left[1-\frac{D_{2} \rho_{2} \zeta_{22} N_{A}^{2}}{M_{2} R T}\right]-\frac{\rho_{1} \zeta_{12}}{M_{2}}}
$$

It is clear from eq 31 that the substitution of $\zeta_{11}$ $=\zeta_{22}=\zeta_{12}=0$ yields the following simplistic relation:

$$
\frac{\zeta_{13}}{\zeta_{23}}=\frac{D_{2}}{D_{1}}
$$

Although the assumption of negligible friction among the solute molecules is questionable, the trend established in eq 32 is intuitively reasonable because the friction coefficient between solute and solvent molecules should be inversely related to the mobility (self-diffusion coefficient) of the solute, at least at high solvent concentrations. Substitution of this model into eqs 24 and 25 reveals that the cross phenomenological coefficients are indeed equal for case 4 . Thus, this model is consistent with the Onsager relations.

\section{Modeling of Drying}

Although the assertions of Onsager may provide valuable guidance, the fundamental basis on

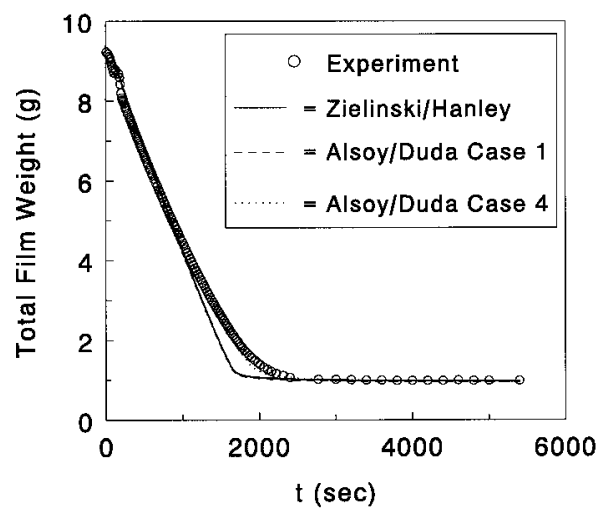

Figure 1. Predictions and experimental drying behavior of a multicomponent polymer solution composed of PS, TL, and EB. Predictions were performed via the insertion of various diffusion models into the multicomponent drying analysis developed by Alsoy and Duda. ${ }^{19}$

which the Onsager relations ${ }^{10}$ were originally developed has been severely criticized, ${ }^{16,17}$ in which case these relations may be more accurately considered postulates, open to experimental confirmation or disproof, rather than proven theorems. In Rational Thermodynamics, ${ }^{16}$ Truesdell remarks, "Onsager's and Casimir's claims that their assertions follow from the principle of microscopic reversibility have been accepted with little question ... the reversibility theorem and Poincarés recurrence theorem make irreversible behavior impossible for dynamical systems in a classical sense. Something must be added to the dynamics of conservative systems, something not consistent with it, in order to get irreversibility at all." With this thought in mind, we applied each of the models discussed to a polymer drying simulation program to examine their utility in predicting the rate of multicomponent solvent removal as a function of time.

In Figures 1 and 2, we compare experimental drying data for the systems polystyrene (PS) toluene (TL)-ethylbenzene (EB) ${ }^{19}$ and PS-TLtetrahydrofuran (THF), ${ }^{27}$ respectively, to predictions from the drying model developed by Alsoy and Duda. ${ }^{19}$ The predictions are obtained from the various multicomponent diffusion models discussed previously with the appropriate model constants. The ternary self-diffusion coefficients required for use in the multicomponent diffusion models have been predicted from free-volume correlations of binary diffusion data, whereas the thermodynamic terms have been evaluated from the ternary extension of Flory-Huggins theory with interaction parameters evaluated from bi- 


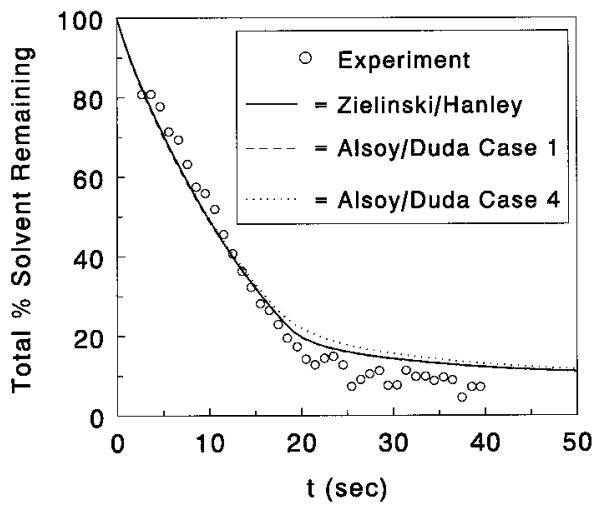

Figure 2. Predictions and experimental drying behavior of a multicomponent polymer solution composed of PS, TL, and THF. Predictions were performed via the insertion of various diffusion models into the multicomponent drying analysis developed by Alsoy and Duda. ${ }^{19}$

nary data. It is important to recognize that the curves displayed in these figures represent a priori predictions and are not merely data correlations. These figures reveal that, in both ternary systems, each of the models performs adequately well and the predictions from the Zielinski-Hanley model and the Alsoy-Duda case 1 model are indistinguishable. Predictions from the case 4 model are noticeably better than those from the case 1 and Zielinski-Hanley models for the PSTL-EB system and slightly poorer for the PSTL-THF system.

Experimental drying data for the system poly(vinyl acetate)-TL-chloroform, accompanied by complementary predictions, ${ }^{28}$ are presented in Figures $3-5$. For this system, the predictions from the Zielinski-Hanley and case 1 models are in excellent agreement with the experimental data, whereas the case 4 model clearly underpredicts the drying rate. Included in Figures 3-5 are predictions from the respective diffusion models using only the principal diffusion coefficients (i.e., the cross diffusion coefficients were set equal to zero, as is common practice). On the basis of the results evident in these figures, the cross diffusion coefficients are clearly not negligible and strongly impact drying rate predictions.

The three examples previously illustrated indicate that inconsistency with the Onsager relations neither nullifies the practical utility of a multicomponent diffusion model nor implies superior performance of those models that are consistent with the OCRs. Our intent here is to identify several multicomponent diffusion models so that their practical utility might be evaluated.

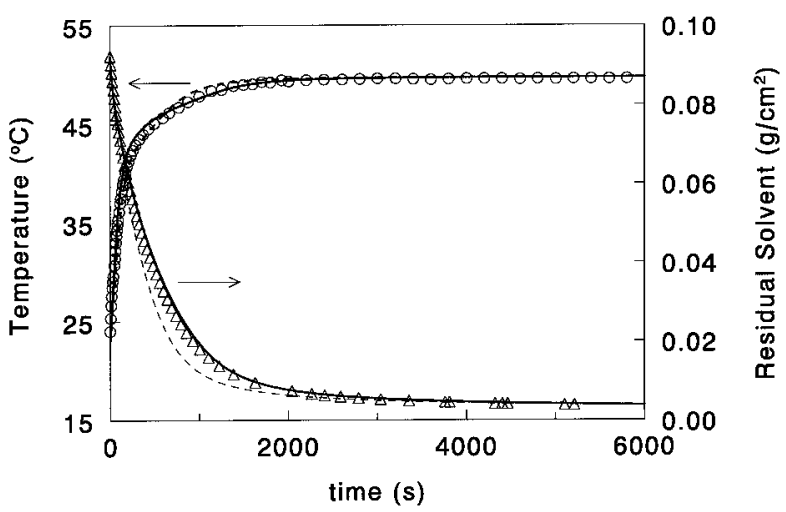

Figure 3. Experimental data and model predictions of the drying behavior of a multicomponent polymer solution composed of poly(vinyl acetate), TL, and chloroform based on the Zielinski-Hanley multicomponent diffusion model. Predictions were performed by the incorporation of the Zielinski-Hanley model into a multicomponent drying analysis developed at $3 \mathrm{M} .{ }^{28}$ The solid line corresponds to the full model, whereas the dashed line corresponds to the solution when the cross coefficients are assumed to be negligible.

Because theoretical development has exceeded the volume of pertinent experimental data currently available, rigorous comparative analysis of existing models in light of the Onsager relations cannot be deemed conclusive. More high-quality data are clearly required before any substantive

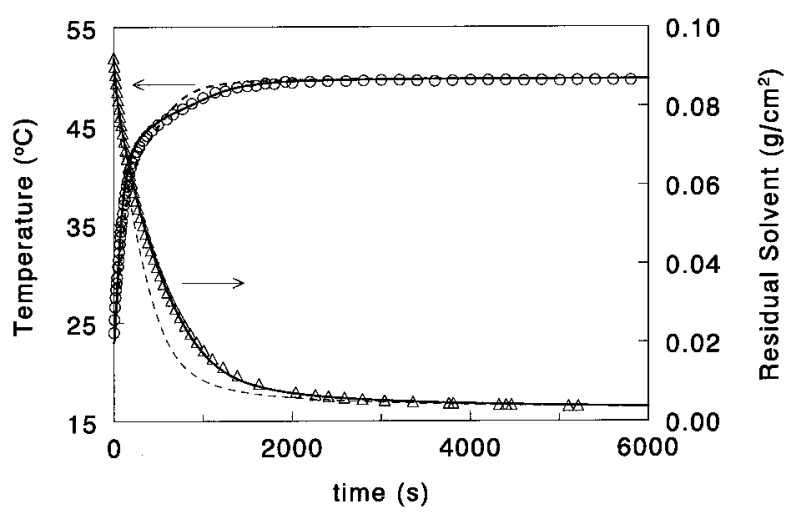

Figure 4. Experimental data and model predictions of the drying behavior of a multicomponent polymer solution composed of poly(vinyl acetate), TL, and chloroform based on the Alsoy-Duda case 1 multicomponent diffusion model. Predictions were performed by the incorporation of the case 1 model into a multicomponent drying analysis developed at $3 \mathrm{M} .{ }^{28}$ The solid line corresponds to the full model, whereas the dashed line corresponds to the solution when the cross coefficients are assumed to be negligible. 


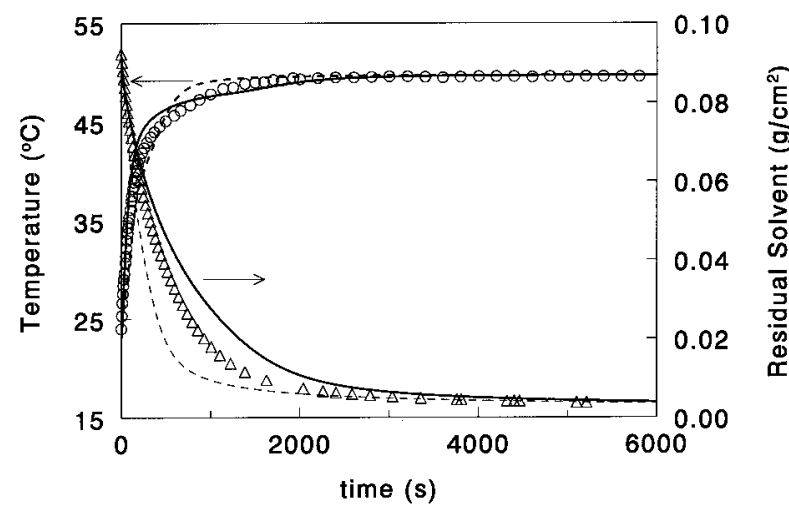

Figure 5. Experimental data and model predictions of the drying behavior of a multicomponent polymer solution composed of poly(vinyl acetate), TL, and chloroform based on the Alsoy-Duda case 4 multicomponent diffusion model. Predictions were performed by the incorporation of the case 4 model into a multicomponent drying analysis developed at $3 \mathrm{M} .{ }^{28}$ The solid line corresponds to the full model, whereas the dashed line corresponds to the solution when the cross coefficients are assumed to be negligible.

progress can be made in terms of discriminating the effectiveness of such models and the correctness of the Onsager relations. In addition, we point out a need for further work in the field of irreversible thermodynamics to elucidate whether systems invariably attempt to minimize entropy production, as initially suggested by Onsager. It is conceivable that systems may behave in a different fashion by, for example, attempting to maximize entropy. ${ }^{17}$ In this scenario, the force driving a system back to equilibrium would be defined as the entropy gradient.

\section{CONCLUSIONS}

We have shown that multicomponent diffusion models can be cast into a generic linear form in terms of chemical potential gradients. The difference between these models is encompassed in the expressions employed in the gradient prefactors. A simple Onsager consistency check derived for the generic form of transport coefficients has been developed and applied to these diffusion models in an attempt to gauge their validity and differentiate between the expressions.

Although these models stem from the Bearman formalism, which is consistent within the context of the Onsager framework, we have determined that certain models obey the Onsager relations, whereas others clearly do not. In three test cases, however, each model is found to represent the multicomponent drying of polymer films reasonably well. These examples illustrate that inconsistency with the Onsager relations neither nullifies the practical utility of a multicomponent diffusion model nor implies superior performance of those models exhibiting consistency. Our intent is to discuss several multicomponent diffusion models so that their practical utility and theoretical validity might be assessed by the research community.

On a larger scale, we note that the Onsager development is considered by many an unproven postulate that is still open to experimental confirmation or disproof. Alternative developments based on irreversible thermodynamics suggest that it may be more appropriate to consider the entropy gradient to be the force driving a system back to a maximum entropy equilibrium state rather than the principal of minimum entropy production championed by Onsager. This work clearly confirms that reliable experimental data are prerequisite in evaluating the rigor of theoretical developments that have never been adequately evaluated but have, over the course of time, been generally accepted without question.

The authors thank Peter Price of 3M Co. for kindly providing them with his experimental data and simulation results for the TL-chloroform-poly(vinyl acetate) system and Dr. Brian Hanley of Jaeger Products for encouraging them to examine the models in terms of the Onsager analysis and for his helpful discussions.

\section{REFERENCES AND NOTES}

1. For example, see Puri, P. S. Gas Sep Purif 1990, 4, 29.

2. For example, see Henis, J. M. S.; Tripodi, M. K. J Membr Sci 1981, 48, 233.

3. Tsay, C. S.; McHugh, A. J. J Polym Sci Part B: Polym Phys 1990, 28, 1327.

4. Shojaie, S. S.; Krantz, W. B.; Greenberg, A. R. J Membr Sci 1994, 94, 255.

5. Shojaie, S. S.; Krantz, W. B.; Greenberg, A. R. J Membr Sci 1994, 94, 281.

6. Cussler, E. L. Multicomponent Diffusion; Elsevier: Amsterdam, 1976.

7. Stefan, J. Wien Ber 1872, 65, 323.

8. Maxwell, J. C. Philos Trans R Soc 1867, 157, 49.

9. Lightfoot, E. N.; Cussler, E. L.; Rettig, R. L. AIChE $\mathrm{J}$ 1962, 8, 702 .

10. Onsager, L. Phys Rev 1931, 37, 405. 
11. Miller, D. G. J Phys Chem 1958, 63, 570.

12. Woolf, L. A.; Miller, D. G.; Gosting, L. J. J Am Chem Soc 1962, 84, 317.

13. Bearman, R. J. J Phys Chem 1961, 1961, 65.

14. De Groot, S. R.; Mazur, P. Non-Equilibrium Thermodynamics; Elsevier: Amsterdam, 1962.

15. Fitts, D. D. Non-Equilibrium Thermodynamics: A Phenomenological Theory of Irreversible Processes in Fluid Systems; McGraw-Hill: New York, 1962.

16. Truesdell, C. A. Rational Thermodynamics, 2nd ed.; Springer-Verlag: New York, 1984, 375.

17. Jaynes, E. T. Ann Rev Phys Chem 1980, 31, 579.

18. Zielinski, J. M.; Hanley, B. F. AIChE J 1999, 45, 1.

19. Alsoy, S.; Duda, J. L. AIChE J 1999, 45, 896.

20. Hartley, G. S.; Crank, J. Trans Faraday Soc 1949, 45,801 .
21. Darken, L. S. Trans Am Inst Mining Met Eng 1948, $175,184$.

22. Loflin, T.; McLaughlin, E. J Phys Chem 1969, 75, 186.

23. McCall, D. W.; Douglass, D. C. J Phys Chem 1967, $71,987$.

24. Zielinski, J. M. J Polym Sci Part B: Polym Phys 1996, 34, 2759.

25. Foley, G.; Cohen, C. J Polym Sci Part B: Polym Phys 1987, 25, 2027.

26. Hess, W.; Nägele, G.; Akcasu, A. Z. J Polym Sci Part B: Polym Phys 1990, 28, 2233.

27. Drake, M. C.; Wang, S. Presented at the AIChE Annual Meeting, San Francisco, CA, 1989.

28. Price, P. E.; Hadj Romdhane, I.; Miller, C. A.; Wang, S. 3M Engineering Systems Technology Center, Unpublished results, 2000. 